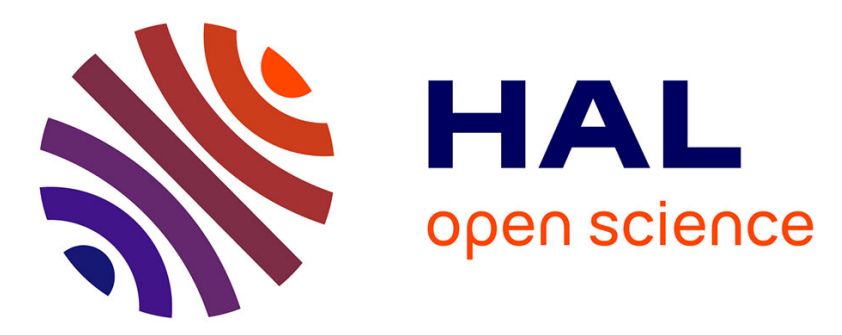

\title{
An ontology for unexpected events and actions in chronic patient home care protocol
}

\author{
Amira Derradji, Agnès Front, Christine Verdier, Vincent Bouzon
}

\section{To cite this version:}

Amira Derradji, Agnès Front, Christine Verdier, Vincent Bouzon. An ontology for unexpected events and actions in chronic patient home care protocol. Tenth International Conference on Research Challenges in Information Science (RCIS 2016), May 2016, Grenoble, France. pp.1 - 6, 10.1109/RCIS.2016.7549317 . hal-01466528

\section{HAL Id: hal-01466528 \\ https://hal.science/hal-01466528}

Submitted on 13 Feb 2017

HAL is a multi-disciplinary open access archive for the deposit and dissemination of scientific research documents, whether they are published or not. The documents may come from teaching and research institutions in France or abroad, or from public or private research centers.
L'archive ouverte pluridisciplinaire HAL, est destinée au dépôt et à la diffusion de documents scientifiques de niveau recherche, publiés ou non, émanant des établissements d'enseignement et de recherche français ou étrangers, des laboratoires publics ou privés. 


\section{An Ontology for Unexpected Events and Actions in Chronic Patient Home Care Protocol}

\author{
Amira Derradji \\ Univ. Grenoble Alpes, LIG \\ Saint Martin d'Hères, France \\ amira.derradji@imag.fr \\ amira.derradji@arcan.fr
}

\author{
Agnès Front \\ Univ. Grenoble Alpes, LIG \\ Saint Martin d'Hères, France \\ agnes.front@imag.fr
}

\author{
Christine Verdier \\ Univ. Grenoble Alpes, LIG \\ Saint Martin d'Hères, France \\ christine.verdier@imag.fr
}

\author{
Vincent Bouzon \\ Arcan Systems \\ Bron, France \\ vincent.bouzon@arcan.fr
}

\begin{abstract}
A health care protocol for a chronic disease contains a set of actions that describe how the patient should treat his/her disease outside the hospital. The patient and possibly his healthcare team should follow the protocol in order to control the evolution of the chronic disease. The main problems are that (i) the patient can't interact and integrate in the protocol the expertise he acquired during his daily life with the disease and (ii) the health care team doesn't know how the protocol is concretely followed. Our main contribution in this paper is an approach for integrating the patient's expertise knowledge expressed in textual or verbal form, and representing this expertise with an ontological paradigm.
\end{abstract}

Index terms - p-health, health care protocol, chronic expert patient, ontology.

\section{INTRODUCTION}

A chronic disease requires long life treatments, which need the integration of patient's care in his own environment outside the hospital (or other institutions) and under continuous supervision by the healthcare team.

Home care for chronic diseases needs to apply continuously a set of actions, called "care protocol", defined by healthcare professionals, related to the disease follow-up, treatment, medication, etc. and indicating the patient (or his caregivers) how to treat and follow the disease.

One problem is the fact that this care protocol is not personalized to patient who often carries out alone several protocol's actions such as the control of vital parameters or some parts of the treatment. Doing that, he develops an important expertise on his chronic disease and can be considered as an "expert patient".

The patient's expertise in daily care offers important benefits to both patient and healthcare professionals, such as the knowledge of the concrete execution of the care protocol, the fact that some actions are not realized or correctly realized or are modified by the patient, and what happens to him as consequences. Designing and implementing these elements are our major challenges.

Moreover, more than continuously assisting the patient, our goal is also to provide healthcare professionals with detailed information about the patient's disease progression. This information can be very useful to discover prejudicial situations that require an intervention of the healthcare team. Indeed, during the disease follow-up, the patient's expertise in daily care can result in (i) actions not correctly made or modified and (ii) events undefined in the initial protocol that may happen. Both of them (unexpected actions and unexpected events) reported by the patient should be sent to the healthcare team who will analyze them and decide which one may be of benefit for the patient's care.

For that purpose, we propose in this paper to use the ontological paradigm that is widely used in healthcare domain. Indeed, ontologies have proved to be powerful in expressing expertise knowledge and represent it from human understandable into machine-readable format. The ontology will allow formalize a chronic patient expertise terminology (particularly chronic disease parameters, unexpected actions and unexpected events) that can be reused in other ontologies in the same domain. We propose to use traditional reasoning techniques and rules that define concepts values and relations between them.

We propose to do the traditional reasoning techniques on ontologies with rules defined on concepts values and on the relations between them. Thus, we propose to model relationships between the ontology concepts and to reason from patient's expertise.

The paper is organized as follows. Section II presents the case study on the chronic disease "type 1 diabetes". Section III presents our general approach. Section IV describes the ontology proposed. Section V presents a survey of related works applying ontologies in medicine and in home care. The conclusion is drawn in the last section.

\section{CASE STUdY}

This section presents a use case underlying our research. This use case is relative to the diabetes. The diabetes disease is a metabolic disorder characterized by the presence of high blood sugar (glucose) level due to defective insulin secretion, defective insulin action or both. There are two major types of diabetes, called type 1 and type 2 . In type 1 , the beta cells of the pancreas do not produce insulin. In type 2, the beta cells of the pancreas do not produce enough insulin for proper function. The patients with diabetes should know particularly how to control the level of blood sugar, how to inject insulin, how to recognize and treat hyperglycemia (high blood sugar level) and how to recognize and treat hypoglycemia (low blood sugar level). 
To answer these questions, healthcare professionals defined a set of actions (the care protocol) that need to be achieved by the patient (or possibly caregivers). For example, the hypoglycemia care protocol for type 1 diabetes can be summarized as follow:

1) The patient recognizes the symptoms (e.g., weakness, headache, hunger, rapid heartbeat, etc.).

2) The patient makes himself safe (e.g., pull over if he is driving, sit down if he is walking, etc.).

3) The patient tests his blood sugar level using blood glucose meter to make sure that he is under an hypoglycemic episode.

4) The patient gets some carbs quickly into his body in order to avoid the side effects of this episode. For that, it is recommended to take 15 or 20 grams of sugar. Some foods can also quickly raise the blood sugar level such as 1 cup of fruit juice, 1 tablespoon of honey, etc.

5) The patient waits 20 to 30 minutes, then he checks his blood sugar level again with blood glucose meter. If it is still low, he eats again 15 or 20 grams of sugar. The patient repeats this action until his blood sugar level is higher than or equal to $70 \mathrm{mg} / \mathrm{dl}$. It is also recommended, once the level is fixed, to eat more carbohydrate such as bread. Sometimes, the hypoglycemic episode is severe and causes the patient's unconsciousness. In this case, the caregiver (or the helper) should give him an emergency glucagon injection that quickly raises blood sugar levels.

6) The patient should look for the causes of hypoglycemic episode and discuss with the doctor about the severe case of hypoglycemia, the inscription of glucagon injection, and the adaptation of his diabetes management plan.

Sometimes, the patient doesn't strictly follow the care protocol. He may skip, modify or replace an action. For example the patient may skip the action (3) and immediately perform the action (4). He may also modify action (4) for example by "taking more sugar than 15 or 20 grams, until the disappearance of hypoglycemia symptoms" or replace this action by another such as "taking emergency glucagon injection". The ontology proposed (cf. section IV) aims to detect these different cases of unexpected events and actions.

\section{THE GENERAL APPROACH}

In previous works [1], we proposed to represent the home care protocol as a set of actions proposed to the patient in a web interface. This protocol is built by a medical team through their own web interface. The "healthcare professionals access" allows healthcare professionals to design, consult and adapt the care protocol.

In this paper, we illustrate the "chronic patient access" that allows a chronic patient (or his caregivers) to consult and follow the care protocol, and to monitor it by recording parameters or reporting unexpected events and actions. The web application interfaces showed in figure 1 present the implementation on a mobile device (e.g., a tablet) of the care protocol to be followed by the patient.

The construction of the care protocol relies on a Domain Specific Language (DSL) for the construction of care protocols

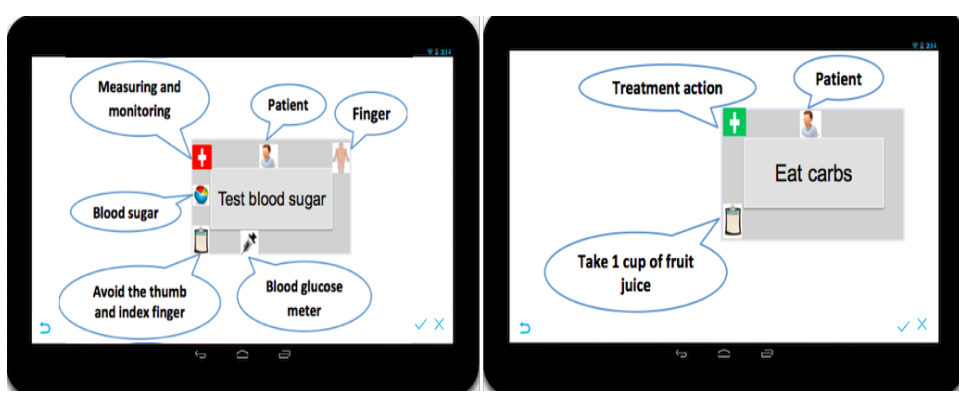

Fig. 1: Patient interaction interface for following the care protocol

that we defined in [1]. This DSL is based on an abstract syntax (meta-model) and a concrete syntax (graphical notation). On one hand, the meta-model describes and formalizes the main concepts of care protocol, which were extracted from a thorough study of various chronic diseases care protocols such as (1) expected actions: treatment action (e.g., inject insulin), measuring and monitoring action (e.g., control the blood sugar level), education action (e.g., wash hands before the blood sugar control) and preventive action (e.g., take the influenza vaccine), (2) actors: patient, healthcare professionals (e.g., diabetologist, nurse) and caregivers (e.g., family, friends), (3) diseases (e.g., type 1 diabetes), (4) drugs (e.g., insulin), medical devices (e.g., blood glucose meter), medical consultation (e.g., consultation with diabetologist), exam/act (e.g., glycated hemoglobin exam), (5) parameters (e.g., blood sugar), (6) good practices (e.g., insulin pen needle are disposable), (7) parts of body (e.g., abdomen) and (8) expected events (e.g., presence of lipodystrophy on the abdomen). On the other hand, a graphical notation is associated with each concept of the meta-model and is represented by clickable information, which provides more information about the concept. For example, in figure 1 (left side), the actor "Patient" should execute the measuring and monitoring action "Test blood sugar" which allows to test the parameter "Blood sugar" with medical device "Blood sugar meter" on the part of body "Finger" and respect the good practice "Avoid the thumb and index finger". Thus, in figure 1 (right side), the actor "Patient" should execute the treatment action "Eat carbs" and respect the good practice "Take 1 cup of fruit juice".

Moreover, a special interface is dedicated to report unexpected event with three main areas: emergency, pain and a dialogue box to express the problem (figure 2). Both, "emergency" and "pain" areas represent respectively the emergency and pain levels estimated by the patient. The emergency level is represented by a six-valued scale, from 0-not urgent to 5extremely urgent. For the pain level, the scale also offers six values, but with different meanings: 0-very moderate and 5very important. Finally, the patient describes the unexpected event in natural language in the corresponding text box.

The general approach of the treatment of the patient interaction is shown in the figure 3 . We propose an ontology to exploit the report of all the unexpected events and actions and 


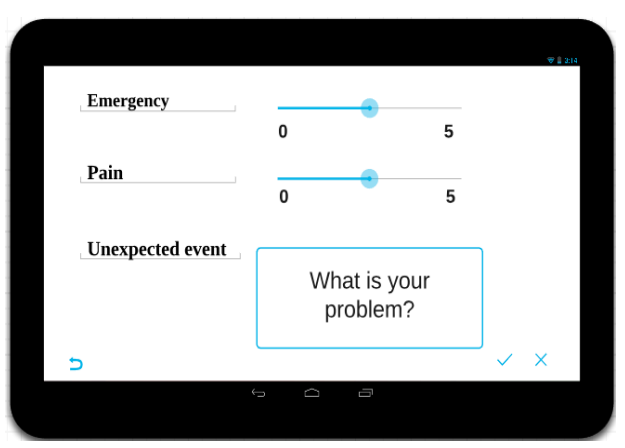

Fig. 2: Patient interaction interface for unexpected event

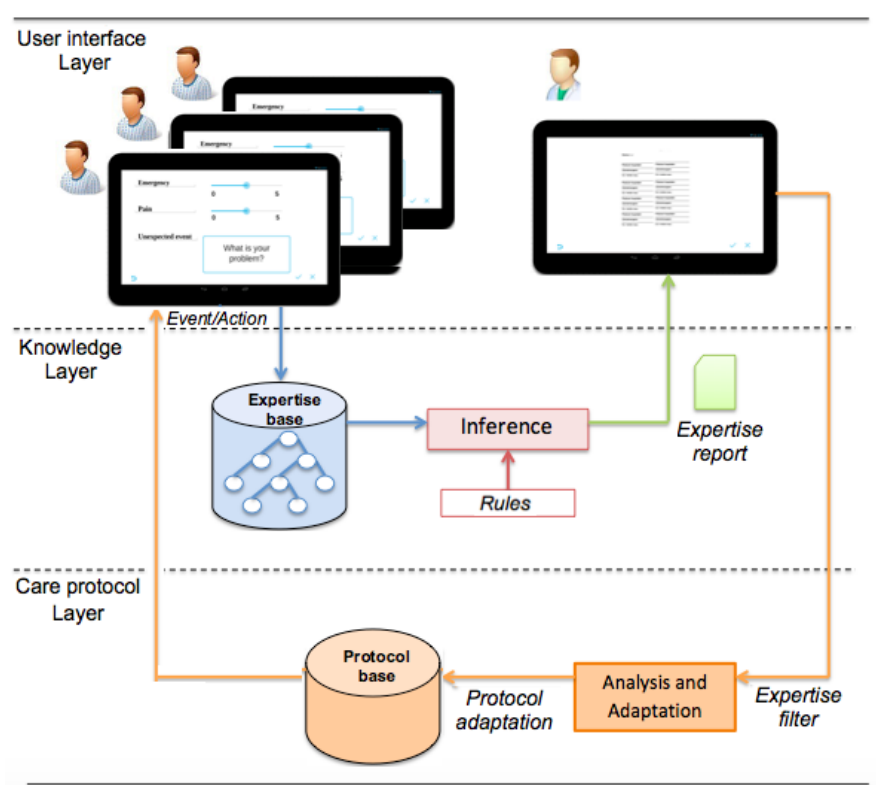

Fig. 3: Treatment of patient interaction

to infer important medical information that could be transferred to healthcare professionals. In particular, a comparison between the "expected action" as defined in the protocol and the "unexpected action" as realized and reported by the patient, needs to be done and analyzed. A report including detailed results will be generated and sent to healthcare professionals who could analyze the result and check the consequences of this action on the patient's health and life's quality.

All unexpected action that could have negative consequences will be filtered. The other unexpected actions will be retained and added to the initial care protocol in order to adapt and adjust the protocol to this new deal. If some events or actions are recurrent and "medically" interesting, it can become a new medical knowledge to be disseminated.

\section{THE KNOWLEDGE LAYER}

This section presents the ECPO (Event Chronic Patient health care Ontology) that we designed for unexpected event and action in home care protocols.

\section{A. Ontology and ontology engineering}

An ontology is defined as a "formal explicit specification of a shared conceptualization" [2]. Four notions are important in this definition. First, conceptualization refers to an abstract model of the world that we wish to represent. Secondly, explicit means that the type of concepts identified, and the constraints of their use, are explicitly defined. Thirdly, formal refers to the fact that the ontology should be machine-readable. Finally, shared means that a ontology captures consensual knowledge, that is not a personal view of the target phenomenon of some particular individual, but one accepted by a group.

An ontology is composed of sets of concepts, relations, attributes and data types. Concepts (or classes) are sets of real world entities with common features (e.g., drug).

Relations are binary associations between concepts. There exists inter-concept relations, which are common to any domain, and domain-dependent associations (e.g., care protocol is composedOf expected action). Attributes represent quantitative and qualitative features of particular concepts (e.g., code of drug), which take values in a given scale defined by the data type (e.g., integer, string, etc.).

Additionally, an ontology can contain instances (or individuals) (e.g., insulin is an instance of the concept drug)

Binary relations can be defined between concepts. In those cases, the concept at the origin of the relation represents the domain and those at the destination, the range. For example the relationship "hasDuration" has the concept "Unexpected event" as domain and the concept "Duration" as range. The relationships may fulfill properties such as symetry or transitivity.

In addition, concepts may represent overlapping sets of real entities (i.e. an individual may be an instance of several concepts simultaneously). In this case, ontology languages allow specify that two or more concepts are disjoint (i.e. individuals can only be instances of one of those concepts).

Some standard languages have been developed to construct ontologies. They are usually based on first-order logic or on description logic. The most used are OWL (Ontology Web Language) [3] and RDF (Resource Description Framework) [4].

\section{B. The ECPO}

In our research, ECPO has been designed with a synthesis of different methodological approaches [5] [6] [7] existing in the literature. This consists in the following steps:

- Specification: consists in identifying the domain covered by the proposed ontology, the purpose, the users and the data sources used to create it. The result of this step is the informal specification requirements document.

- Conceptualization: consists in defining the concepts and relationships between them. The result of this step is the conceptual model of the ontology.

- Implementation: once the conceptual model is defined, it will be encoded using an ontology language and an ontology editor. The result of this step is the ontology. 


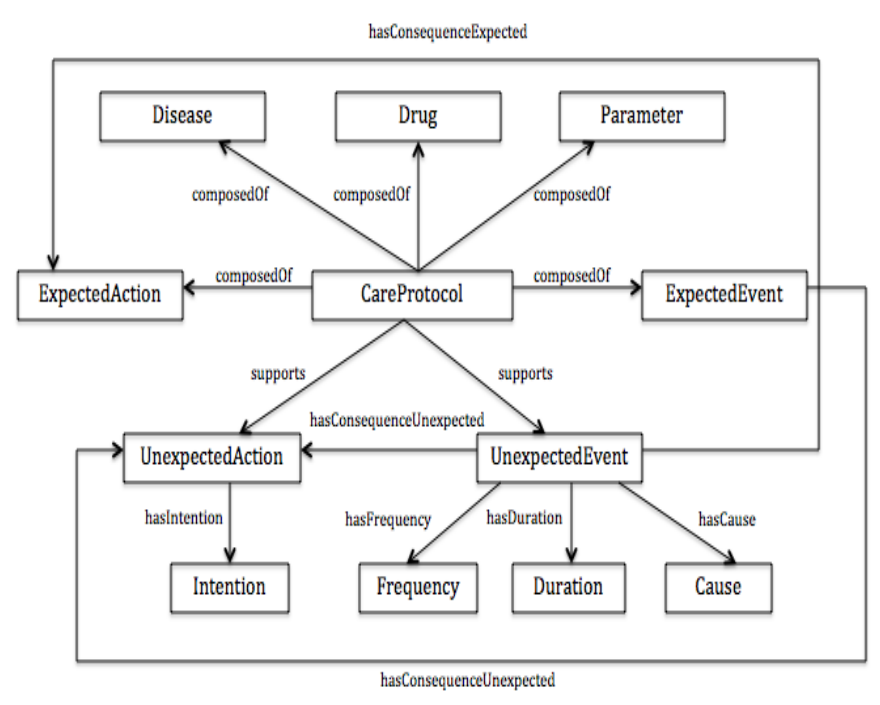

Fig. 4: Main concepts and relationships of ECPO

- Evaluation: after the development of the ontology, an evaluation will be done using an ontology reasoner in order to detect pitfalls.

1) Specification: This subsection details the first step of the ontology development which identifies:

- Domain of the ontology: chronic patient care protocol.

- Purpose of the ontology: allow healthcare professionals to interpret concretely the unexpected events or the unexpected actions done by chronic patient during the protocol application.

- Users of the ontology: the healthcare professionals, the helpers and the patient.

- Data sources: medical data about chronic diseases and care protocol.

2) Conceptualization: This step is the most important step in an ontology development. It consists in the definition of the content of the ontology. The proposed ontology ECPO includes the necessary concepts related to unexpected events and actions in a care protocol.

Figure 4 illustrates the main concepts and relationships of the proposed ontology ECPO. Boxes represent concept and lines represent relationships.

The main concepts composing this ontology are:

1) CareProtocol: is the chronic disease care protocol defined by the healthcare team which describes what the patient needs for monitoring his disease outside the hospital (cf. section III). The following relationships are defined for a CareProtocol:

- composedOf: CareProtocol $\rightarrow$ Disease, represents the disease associated with the care protocol.

- composedOf: CareProtocol $\rightarrow$ Drugs, represents the drug associated with the care protocol.

- composedOf: CareProtocol $\rightarrow$ Parameter, represents the parameter associated with the care protocol.
2) ExpectedAction: is a care protocol's action defined by the healthcare team which describes how the patient should treat his disease outside the hospital. The following relationship is defined for an ExpectedAction:

- composedOf: CareProtocol $\rightarrow$ ExpectedAction, represents actions to be executed in care protocol.

3) ExpectedEvent: represents a known occurrence defined in the initial care protocol. An expected event describes what may happen to the patient. The following relationships are defined for an ExpectedEvent:

- composedOf: CareProtocol $\rightarrow$ ExpectedEvent, represents the expected event that may occur in a care protocol.

- hasConsequenceUnexpected: ExpectedEvent $\rightarrow$ UnexpectedAction, associates an excepted event to an undefined action performed by the patient.

4) UnexpectedEvent: represents an undefined occurrence for the patient that may happen during his daily life. The following relationships are defined for an UnexpectedEvent:

- hasCause: UnexpectedEvent $\rightarrow$ Cause, is the reason why the unexpected event happens. The cause is given according to the patient's point of view, it may be correct or incorrect.

- hasDuration: UnexpectedEvent $\rightarrow$ Duration, is the period during which the unexpected event occurred.

- hasFrequency: UnexpectedEvent $\rightarrow$ Frequency, is the number of times that the unexpected event happens during daily care protocol.

- has ConsequenceUnexpected: UnexpectedEvent $\rightarrow$ UnexpectedAction, indicates that the patient may perform an unexpected action as consequence of an unexpected event.

- hasConsequenceExpected:

UnexpectedEvent $\rightarrow$ ExpectedAction, indicates that the patient may perform an excepted action as consequence of an unexpected event.

- supports: CareProtocol $\rightarrow$ UnexpectedEvent, represent the integration of all unexpected events in the initial care protocol. This integration is possible after the decision of healthcare professionals.

5) UnexpectedAction: an action performed by the patient that is undefined in the care protocol. The following relationships are defined for the UnexpectedAction:

- hasIntention:UnexpectedAction $\rightarrow$ Intention, describes the patient's determination to perform the unexpected action.

- supports: CareProtocol $\rightarrow$ UnexpectedAction, represents the integration of all unexpected actions in the initial care protocol after their analysis and validation by the healthcare professionals.

The relationship between ExpectedEvent and ExpectedAction is not considered by ECPO because this relationship is defined in the initial care protocol and our proposed ontology is interested in cases of unexpected event or unexpected action or both.

In addition to these concepts and relationships between them, 
we defined some instances of the concepts in the ontology, for example:

- Unexpected event: have vomiting.

- Cause: overdose of a drug (Metformin $850 \mathrm{mg}$ ).

- Frequency: two times.

- Duration: two hours.

- Unexpected action: take anti-vomiting (Métopimazine 7,5 $\mathrm{mg}$ ).

- Intention: avoid further vomiting.

3) Implementation: This section describes the ECPO implementation. For that, we used the ontology language OWL-DL [8] and the open source ontology editor Protégé [9].

OWL-DL allows high level of expressiveness based on description logic and checks the inconsistency of the ontology using automatic reasoning. On the other hand, the open-source ontology editor Protégé is the most widely used tool for ontology development. Protégé allows the construction of ontologies in a simple and intuitive way and the generation of OWL code.

The ECPO is displayed in figure 5 using OWLViz plugin by Protégé.

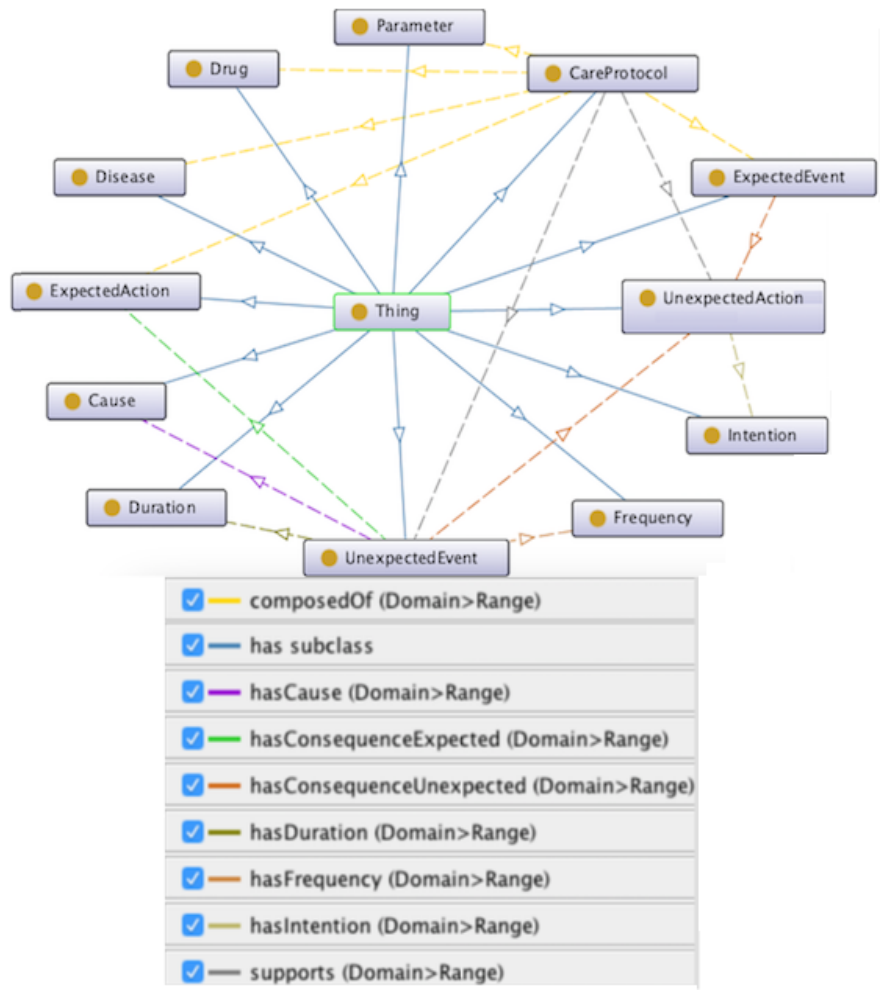

Fig. 5: ECPO in Protégé

4) Evaluation: After the development of the ECPO, the final step is conducted in two parts. On the one hand, the ontology consistency was evaluated via a reasoner in order to test the modelled knowledge of our proposed ontology. For that, we used Pellet ${ }^{1}$ as a reasoner during the ontology development

${ }^{1}$ http://clarkparsia.com/pellet process. On the other hand, the ontology will be tested by using SPARQL rules to identify the unexpected events and the unexpected actions. In order to validate this second part of the evaluation, we proposed some concrete scenarios related with the patient's expertise.

- Scenario 1: the patient forgets to take a dose of a drug at lunch (unexpected event), so he decides to take two doses of this drug at dinner (unexpected action).

- Scenario 2: the patient is under an hypoglycemic episode (expected event), he eats sugar until the disappearance of hypoglycemia symptoms (and not 15 or 20 grams of sugar as indicated by the medical team (unexpected action) in order to avoid repetitive hypoglycemia episodes (intention). He thinks that an intense physical exercise is the cause of this episode (cause).

- Scenario 3: the patient has vomiting (unexpected event) during two hours (duration) when he takes too much a drug (cause), so he decides to take an anti-vomiting (expected action).

- Scenario 4: the patient has headache (unexpected event) for which he thinks the injection of insulin is the cause (cause).

- Scenario 5: the patient takes two fruits before exercise (unexpected action) to avoid a malaise during exercise (intention).

The formalization of SPARQL rules and the test of these five scenarios are part of our future works.

\section{RELATED WORKS}

This section presents the related works employing ontologies in medecine and home care.

\section{A. Ontologies in medecine}

The use of ontologies in medicine has been widely adopted. Some ontologies are implemented to represent taxonomies of medical concepts: for example, the taxonomy for health information technology terms [10], the taxonomy for the treatment of hypertension [11] or the Health Terminology/Ontology Portal (HeTOP) [12].

Some ontologies are proposed in order to manage clinical guidelines. For example, in [13], the authors design with OWL, concepts and relationships involved in a clinical guideline. In the same context, [14] proposes Context-Task Ontology (CTO) that contains the knowledge needed in the development of clinical guidelines.

There are other ontologies focus on the management of pathologies, for example, the ontology for the management of hypertension [15] and the ontology for pneumology [16].

\section{B. Ontologies in home care}

Ontologies have also been used in home care domain in order to represent and share a structured and well-defined terminology between different actors involved in health care. Some examples of ontologies focused on home care domain are Case Profile Ontology (CPO) and Actor Profile Ontology (APO) resulting from the K4Care project [17]. This project aimed to 
develop a web platform that delivers a set of home care services to all actors involved in home care for elderly patients. In this work, both ontologies are encoded in OWL-DL with Protégé and are built following the method On-To-Knowledge. CPO [18] describes home care medical concepts integrating diseases, symptoms, syndromes, clinical intervention, assessment tests, laboratory analysis and social issues. On the other hand, APO [19] is based on actors and includes the necessary knowledge related to the home care of elderly patients such as actors roles, actions, services, documents. Related with the same context, another ontology called OntoPAD [20] is proposed in the ABAH (Agent-Based Architecture for a Cooperative Information System) project. OntoPAD describes actors, actions and documents used in home care. In [21], authors propose an ontology to represent health monitoring and alerting in chronic patient home care systems.

\section{Discussion}

Several researchers show the successful use of ontologies in the medical domain. Ontologies are used to structure the medical terminology about diseases or medical procedures, the home care for the representation of actors and interventions and the monitoring of chronic patients. More relative to our research, a few ontologies are related with home care domain and some of these ontologies are considered as a standard. Additionally, those ontologies represent and share medical knowledge about diseases, actors, organisation of medical intervention, etc. but neither consider the knowledge related with the daily realization of chronic patient care protocol at home, such as unexpected event (e.g., allergic reaction, missing drugs) or unexpected action (e.g., a diabetic patient eats $20 \mathrm{~g}$ of sugar before sports while the quantity recommended by his doctor is $10 \mathrm{~g}$ ). In our research, we consider the patient as a mine of knowledge insufficiently exploited until now in home care. So, there is a need to exploit this patient's expertise in order to improve his care protocol and this is one of our challenges. Compared to these ontologies, the ECPO ontology aims to:

- Define and formalize knowledge about patient's expertise.

- Access to this knowledge.

- Store and reuse this knowledge.

- Get reasonning about patient's expertise and infer new expertise using properties and rules.

In the next section, we present our findings and conclude with future works.

\section{CONCLUSIONS}

In order to integrate the chronic patient's expertise acquired during the daily care protocol application, we propose an approach based on an ontology to represent the expertise's knowledge. The proposed ontology ECPO allows to identify in a concrete way the unexpected events and the unexpected actions done by the patient and helps the healthcare professionals to take decisions about the patient care protocol according to the ontology results.

As future works, the use of SPARQL rules will be considered, in order to determine prejudicial situations or good practices. In addition, we will focus on combine ontology with the patient's interface to provide expertise reports based on text or speech processing.

\section{REFERENCES}

[1] A. Derradji, "Un méta-modèle et un langage orientés patient pour la représentation de protocoles de soins auto-adaptatifs," in Actes du XXXIIIème Congrès INFORSID, Biarritz, France, May 26-29, 2015, pp. $167-182$.

[2] R. Studer, V. R. Benjamins, and D. Fensel, "Knowledge engineering: principles and methods," Data \& knowledge engineering, vol. 25, no. 1, pp. 161-197, 1998.

[3] W3C-OWL-Group, "Owl 2 web ontology language document overview," 2012. [Online]. Available: https://www.w3.org/TR/owl2-overview/

[4] D. Brickley and R. V. Guha, "Rdf vocabulary description language 1.0: Rdf schema," 2004. [Online]. Available: https://www.w3.org/TR/2004/ REC-rdf-schema-20040210/

[5] J. M. Gómez-Pérez and C. Ruiz, "Ontological engineering and the semantic web," in Advanced Techniques in Web Intelligence-I. Springer, 2010, pp. 191-224.

[6] S. Staab, R. Studer, H.-P. Schnurr, and Y. Sure, "Knowledge processes and ontologies," IEEE Intelligent systems, no. 1, pp. 26-34, 2001.

[7] N. F. Noy, "Ontology development 101: A guide to creating your first ontology: Knowldege systems laboratory, stanford university," Stanford Knowledge Systems Laboratory Technical Report KSL-01-05 and Stanford Medical Informatics Technical Report SMI-2001-0880, 2001.

[8] J. Pulido, M. Ruiz, R. Herrera, E. Cabello, S. Legrand, and D. Elliman, "Ontology languages for the semantic web: A never completely updated review," Knowledge-Based Systems, vol. 19, no. 7, pp. 489-497, 2006.

[9] N. F. Noy, R. W. Fergerson, and M. A. Musen, "The knowledge model of protege-2000: Combining interoperability and flexibility," in Knowledge Engineering and Knowledge Management Methods, Models, and Tools. Springer, 2000, pp. 17-32.

[10] B. E. Dixon, A. Zafar, J. J. McGowan et al., "Development of a taxonomy for health information technology," in Medinfo 2007: Proceedings of the 12th World Congress on Health (Medical) Informatics; Building Sustainable Health Systems. IOS Press, 2007, p. 616.

[11] T. Mabotuwana and J. Warren, "An ontology-based approach to enhance querying capabilities of general practice medicine for better management of hypertension," Artificial Intelligence in Medicine, vol. 47, no. 2, pp 87-103, 2009.

[12] J. Grosjean, T. Merabti, N. Griffon, B. Dahamna, and S. J. Darmoni, "Teaching medicine with a terminology/ontology portal," Stud Health Technol Inform, vol. 180, pp. 949-53, 2012.

[13] A. Kumar, S. Quaglini, M. Stefanelli, P. Ciccarese, and E. Caffi, "Modular representation of the guideline text: An approach for maintaining and updating the content of medical education," Medical informatics and the Internet in medicine, vol. 28, no. 2, pp. 99-115, 2003.

[14] Y. Ye, Z. Jiang, X. Diao, D. Yang, and G. Du, "An ontology-based hierarchical semantic modeling approach to clinical pathway workflows," Computers in Biology and Medicine, vol. 39, no. 8, pp. 722 - 732, 2009.

[15] O. Steichen, C. Daniel-Le Bozec, M.-C. Jaulent, and J. Charlet, "Construction d'une ontologie pour la prise en charge de l'hypertension artérielle," in 18es Journées Francophones d'Ingénierie des Connaissances, 2007.

[16] A. Baneyx, "Construire une ontologie de la pneumologie aspects théoriques, modèles et expérimentations," Ph.D. dissertation, Université Pierre et Marie Curie-Paris VI, 2007.

[17] A. Valls, K. Gibert, D. Sánchez, and M. Batet, "Using ontologies for structuring organizational knowledge in home care assistance," international journal of medical informatics, vol. 79, no. 5, pp. 370-387, 2010.

[18] D. Riaño, F. Real, F. Campana, S. Ercolani, and R. Annicchiarico, "An ontology for the care of the elder at home," in Artificial intelligence in medicine. Springer, 2009, pp. 235-239.

[19] K. Gibert, A. Valls, and J. Casals, "Enlarging a medical actor profile ontology with new care units," in Knowledge Management for Health Care Procedures. Springer, 2008, pp. 101-116.

[20] S. Khalfi, "Construction d'une ontologie pour la prise en charge des patients à domicile," Thèse de Magister en Informatique, Algérie, 2009.

[21] F. Paganelli and D. Giuli, "An ontology-based context model for home health monitoring and alerting in chronic patient care networks," in null. IEEE, 2007, pp. 838-845. 